ISSN 0103-8478

\title{
Farmacocinética do propofol em nanoemulsão em gatos
}

\author{
Pharmacokinetic of propofol in nanoemulsion in cats
}

\author{
Martielo Ivan Gehrcke ${ }^{\mathrm{I}}$ Rafael Messias Luiz ${ }^{\mathrm{I}}$ Marcos Paulo Antunes de Lima ${ }^{\mathrm{I}}$ \\ Felipe Hertzing Farias ${ }^{\mathrm{I}}$ Anicleto Poli ${ }^{\mathrm{II}}$ Aury Nunes de Moraes $^{\mathrm{I}}$ Nilson Oleskovicz ${ }^{\mathrm{I}}$
}

\section{RESUMO}

Os felinos são deficientes na biotransformação do propofol e os dados em relação à farmacocinética nessa espécie são escassos. O objetivo deste estudo foi determinar o perfil farmacocinético da infusão contínua de propofol em nanoemulsão juntamente com a emulsão lipídica em felinos. Utilizaram-se seis gatos sem raça definida (SRD), adultos, machos, castrados, com peso médio de 4,2士0,8kg, em estudo aleatório e de autocontrole. Os animais receberam $10 \mathrm{mg} \mathrm{kg}^{-1} \mathrm{~min}^{-1}$ de propofol a $1 \% \mathrm{em}$ emulsão lipídica (EMU) ou em nanoemulsão (NANO) durante 30 segundos e, imediatamente após, iniciou-se a infusão de 0,3 $m g \mathrm{~kg}^{-1} \mathrm{~min}^{-1}$ da mesma formulação durante 60 minutos. Após 15 dias, receberam o mesmo tratamento com a formulação oposta. Amostras de $3 \mathrm{~mL}$ de sangue venoso foram coletadas nos tempos 0 (basal), 2, 5, 10,15, 30 e 60 minutos de infusão e aos $5,10,15,30,60,90,120,180,240,360,600$ e 1440 minutos após o final da infusão. Os parâmetros farmacocinéticos foram determinados a partir da curva de decaimento da concentração plasmática versus tempo ao final da infusão. A análise estatística foi realizada através de ANOVA-RM com posterior teste t pareado entre os grupos. Não houve diferença entre as formulações em relação a todos os parâmetros. Os volumes de distribuição foram altos com Vdss de 23,23 $\pm 12,30$ litros $\mathrm{kg}^{-1}$ para a nanoemulsão e de 18,12 $\pm 8,54$ litros $\mathrm{kg}^{-1}$ para a emulsão lipídica. Os Cls foram baixos com um $\mathrm{Cl}$ central de 22,20 $\pm 10,83 \mathrm{~mL} \mathrm{~kg}^{-1} \mathrm{~min}^{-1}$ para a nanoemulsão e de $23,42 \pm 13,50 \mathrm{~mL} \mathrm{~kg}^{-1} \mathrm{~min}^{-1}$ para emulsão lipídica. Conclui-se que a farmacocinética do propofol em gatos após infusão contínua caracteriza-se por uma ampla distribuição tecidual e uma lenta eliminação, com possível efeito cumulativo. A formulação em nanoemulsão apresenta características farmacocinéticas semelhantes às da emulsão lipídica.

Palavras-chave: anestesia total intravenosa, formulação, nanotecnologia, infusão contínua, emulsão lipídica.

\section{ABSTRACT}

Cats are deficient in the metabolism of propofol and the data on the pharmacokinetics in this species are scarce. The aim of this study was to determine the pharmacokinetic profile of continuous infusion of propofol in lipid emulsion and compare with the nanoemulsion formulation, in cats. Domestic cats, short hair, adults, male, castrated, weighting $4.2 \pm 0.8 \mathrm{~kg}$ in a randomized and self control trial were used. The animals received $10 \mathrm{mg} \mathrm{kg}^{-1}$ of $1 \%$ propofol in lipid emulsion or nanoemulsion for 30 seconds and immediately after that, a continuous rate infusion of $0.3 \mathrm{mg} \mathrm{kg}^{-1}$ $\mathrm{min}^{-1}$ of the same formulation was administered for 60 minutes. After 15 days the cats received the same treatment with the opposite formulation. Samples of $3 m L$ of venous blood were collected by a central venous catheter inserted in the jugular vein at 0 (baseline), $2,5,10,15,30$, and 60 minutes of infusion and at 5, 10, 15, 30, $60,90,120,180,240,360,600$ and 1440 minutes after the end of the infusion. The pharmacokinetic parameters were determined from the decay curve of plasma concentration versus time at the end of the infusion. Statistical analysis was performed using RMANOVA with subsequent paired t-test between groups. There was no difference between the formulations with respect to all parameters. The volumes of distribution were high with Vdss of $23.23 \pm 12.30$ liters $\mathrm{kg}^{-1}$ for the nanoemulsion and 18.12 \pm 8.54 liters $\mathrm{kg}^{-1}$ for lipid emulsion. The Cls were low with a Cl central to $22.20 \pm 10.83 \mathrm{~mL}$ $\mathrm{kg}^{-1} \mathrm{~min}^{-1}$ for the nanoemulsion and $23.42 \pm 13.50 \mathrm{~mL} \mathrm{~kg}^{-1} \mathrm{~min}^{-1}$ for lipid emulsion. The conclusion is that the pharmacokinetics of propofol in cats after infusion is characterized by a broad tissue distribution and a slow elimination, with possible cumulative effect. The formulation nanoemulsion has pharmacokinetic properties similar to the lipid emulsion.

Key words: total intravenous anesthesia, formulation, nanotechnology, continuous infusion, lipid emulsion.

'Departamento de Medicina Veterinária, Centro de Ciências Agroveterinárias (CAV), Universidade do Estado de Santa Catarina (UDESC), Av. Luiz de Camões 2090, Conta Dinheiro, 88520-000, Lages, SC, Brasil. E-mail: a2no@ cav.udesc.br. *Autor para correspondência. "Departamento de Farmacologia, Campus Universitário Trindade, Universidade Federal de Santa Catarina (UFSC), Florianópolis, SC, Brasil. 


\section{INTRODUÇÃO}

O propofol é um anestésico intravenoso amplamente utilizado na Medicina Humana e Veterinária para indução e manutenção anestésica. Atualmente, sua formulação é composta de óleo de soja, fosfatídeos de ovo purificados e glicerol, formando uma emulsão lipídica, que pode acarretar em lipemia, risco de embolismo e até mesmo contaminações bacterianas (BAKER \& NAGUIB, 2005). Por isso, novas formulações em nanoemulsões estão sendo propostas com intuito de eliminar os veículos e garantir maior estabilidade e segurança na administração (KIM et al., 2007; LEE et al., 2009; CLEALE et al., 2009; TAMANHO, 2010).

Nanoemulsões são definidas como emulsões óleo em água, cujas partículas não ultrapassam 1000 nanômetros, sendo que para o propofol não passam de 100 nanômetros (DATE \& NAGARSENKE, 2008), embora alguns estudos ainda utilizem o termo microemulsão para estas (MOREY et al., 2006; KIM et al., 2007; DATE \& NAGARSENKE, 2008; LEE et al., 2009). São caracterizadas por um núcleo lipídico em que se dissolve o fármaco delimitado por uma camada monomolecular de fosfolipídios, dando à amostra um aspecto translúcido, sem a necessidade de veículos adicionais (BAKER \& NAGUIB, 2005). Entretanto, existem diferentes nanoemulsões que podem ser realizadas devido aos diferentes surfactantes existentes, cada qual com suas características particulares que podem alterar a liberação do princípio ativo e consequentemente o tempo de exposição ao fármaco e suas propriedades farmacológicas (BAKER \& NAGUIB, 2005; DATE \& NAGARSENKE, 2008).

Os felinos são naturalmente deficientes em biotransformar compostos fenólicos como o propofol, o que pode acarretar em efeito cumulativo e atraso na recuperação anestésica (COURT \& GREENBLATT, 2000; PASCOE et al., 2006). Quanto à farmacocinética do propofol nessa espécie, encontram-se apenas os estudos de BESTER (2009) e CLEALE et al. (2009), que utilizaram a formulação em emulsão lipídica e em nanoemulsão, respectivamente, ambos após administração em bolus. Quanto à farmacocinética do propofol em felinos sob infusão contínua, não se encontram dados na literatura, mesmo sabendo-se que um período de infusão melhor determina a fase de equilíbrio e as transferências nos diversos compartimentos (ADAM et al., 1980). Ainda, o estudo de CLEALE et al. (2009) não determina o perfil farmacocinético completo com análise compartimental do propofol nessa espécie.
O objetivo deste estudo foi determinar o perfil farmacocinético completo do propofol em nanoemulsão em gatos sob infusão contínua e compará-lo ao perfil farmacocinético da emulsão lipídica atualmente disponível.

\section{MATERIAL E MÉTODOS}

Foram utilizados seis felinos, machos, com idade entre 2 e 4 anos, sem raça definida (SRD), castrados, com peso médio de $4,2 \pm 0,8 \mathrm{~kg}$ e comprovadamente hígidos após exames clínico e laboratorial (hemograma, perfil renal e hepático completos). Antecedendo o período de experimentação, os animais foram submetidos a jejum alimentar de 10 horas, sem restrição hídrica.

No dia do estudo, foram induzidos à anestesia geral com isofluorano a $5 \mathrm{~V} \%$, administrado via máscara facial conectada a um sistema sem reinalação de gases (Baraka), utilizando-se, como gás diluente, o oxigênio a 100\%. Após, foram mantidos em anestesia com isofluorano a $1,5 \mathrm{~V} \%$ via máscara facial durante 10 a 15 minutos para cateterização das veias jugular direita com cateter venoso central 22G para coleta de amostras sanguíneas e cefálica esquerda, com cateter venoso periférico $22 \mathrm{G}$ para administração dos tratamentos. Após o término do período de instrumentação, a anestesia inalatória foi cessada e aguardou-se 60 minutos para a recuperação total do paciente.

Os animais foram submetidos a um estudo aleatório e de autocontrole, se permitido 15 dias de intervalo entre os tratamentos. Eles foram alocados em dois grupos: nanoemulsão (NANO, $\mathrm{n}=6$ ), que receberam como agente indutor propofol em nanoemulsão (Propovet ${ }^{\circledR}$, Ouro Fino Saúde Animal Ltda, Lote Piloto 001/2009, 10 $\mathrm{mg} \mathrm{mL}^{-1}$ ), que possui registro de patente (CRISTÁLIA PRODUTOS QUÍMICOS FARMACÊUTICOS LTDA, 2008) e já está aprovada para uso em humanos sendo que, em Medicina veterinária, esta encontra-se em vias finais de aprovação junto ao Ministério da Agricultura, Pecuária e Abastecimento (MAPA); e emulsão lipídica (EMU, n=6), que receberam propofol em emulsão lipídica (Propovan ${ }^{\circledR}$, Cristália Produtos Químicos Farmacêuticos Ltda, $10 \mathrm{mg} \mathrm{mL}^{-1}$ ), ambos na dose de $10 \mathrm{mg} \mathrm{kg}^{-1}$ por via intravenosa, segundo estudos prévios de TAMANHO (2010), padronizando-se a velocidade de administração do bolus em 30 segundos, por meio de bomba de infusão. Ato contínuo, iniciou-se a infusão contínua de propofol, na respectiva formulação, na taxa de $0,3 \mathrm{mg}$ $\mathrm{kg}^{-1} \min ^{-1}$ durante 60 minutos. Durante a infusão, 
os animais foram posicionados em decúbito lateral esquerdo sobre colchão térmico para a manutenção da temperatura corporal entre 37 e $38 \mathrm{C}^{\circ}$, sendo a temperatura da sala padronizada entre 22 e $24^{\circ} \mathrm{C}$.

Para análise das concentrações plasmáticas, foram coletadas, em tubos com EDTA, amostras de 3,0mL de sangue venoso por meio do cateter venoso central, anteriormente inserido na veia jugular, retirando-se previamente $1 \mathrm{~mL}$ de amostra de descarte para evitar possíveis diluições. Para cada $3,0 \mathrm{~mL}$ de sangue retirado, foram infundidos, juntamente com o descarte, 3,0mL de solução fisiológica $(\mathrm{NaCl}$ 0,9\%), com o objetivo de manter o volume circulante, sendo o cateter preenchido com $0,3 \mathrm{~mL}$ de solução heparinizada $5 \mathrm{UI} \mathrm{mL} \mathrm{m}^{-1}$. As amostras foram então centrifugadas por 10 minutos a 3500rpm, sendo o plasma alocado em triplicatas e congelado a $-20^{\circ} \mathrm{C}$ por 24 horas, com posterior conservação a $-80^{\circ} \mathrm{C}$ até a análise.

Os tempos de coleta foram: antes da indução (M0) e aos 2, 5, 10, 15, 30 e 60 minutos após o início da infusão contínua. Aos 60 minutos, encerrou-se a infusão e foram coletadas amostras aos $5,10,15,30,60,90,120,180,240,360,600$ e 1440 minutos após o final da infusão.

As concentrações plasmáticas de Propofol foram determinadas por cromatografia líquida (LC) com detecção por Espectofotometria de Massas (MS/MS), utilizando como fonte de ionização a eletronebulização de modo positivo, segundo técnica desenvolvida e validada pelo laboratório BIOTEC (Biotecnologia, Campinas-SP), em que a fase móvel era composta por Fase A: água + 0,5\% de Ác. Fórmico e Fase B: Acetonitrila + 0,5\% de Ác. Fórmico; a coluna analítica Phenomenex Luna C-18 $15 \mathrm{~cm}$; o volume de injeção de $20 \mu \mathrm{L}$ e fluxo de $1 \mathrm{ml}$ $\min ^{-1}$; tempo total da corrida de 7 minutos e limite inferior de quantificação de $20,04 \mathrm{ng} \mathrm{mL}^{-1}$.

Com auxílio do programa Pharmakit ${ }^{\circledR}$ versão 2.10, foram determinados os interceptos das concentrações no eixo y (A, B e C) e macroconstantes de distribuição $(\alpha)$ e de eliminação rápida e lenta $(\beta$ e $\gamma)$, com base em modelo farmacocinético tricompartimental $\left(\mathrm{Cp}=\mathrm{Ae}^{-\alpha t}+\mathrm{Be}^{-\beta t}+\mathrm{Ce}^{-\gamma t}\right)$. Ainda, o software forneceu os valores meia vida de distribuição $\left(\mathrm{t}^{1} / 2 \alpha\right)$ e meias vidas de eliminação rápida e lenta $\left(\mathrm{t}^{1} / 2 \beta\right.$ e $\left.\mathrm{t}^{1} / 2 \gamma\right)$.

A partir desses dados, utilizou-se uma tabela de conversão de dados farmacocinéticos fornecida pelo professor Dr. Shafer da Universitade de Stanford, através do site <http://anesthesia. stanford.edu/pkpd/_layouts/viewlsts.aspx $>$, para cálculo dos valores de: clearance central (Cl1) e intercompartimentais $(\mathrm{Cl} 2$ e $\mathrm{Cl} 3)$, volumes de distribuição central (V1) e periféricos (V2 e V3), volume de distribuição no estado de equilíbrio steady state (Vdss) e as microconstantes de eliminação (k10) e de transferência intercompartimental (k12, k21, k13 e k31).

A análise estatística foi realizada com auxílio do programa GraphPrisma ${ }^{\circledR} 5.0$ por meio de análise de variância para amostras repetidas (ANOVA$\mathrm{RM}$ ), seguida do teste t-pareado para detecção de diferenças entre as formulações. Diferenças foram consideradas significativas quando $\mathrm{P} \leq 0,05$.

\section{RESULTADOS E DISCUSSÃO}

As concentrações plasmáticas permaneceram estáveis em ambos os grupos durante a infusão (Figura 1), não diferindo entre estes, entretanto, a grande variação individual encontrada reflete a utilização de animais de raças mistas, como já relatado na literatura em gatos (BESTER, 2009) e em cães (NOLAN \& REID, 1993) e também em relação a variações individuais dentro da mesma espécie e raça. Também puderam ser evidenciados em alguns animais alguns picos plasmáticos após o início da infusão entre 5 e 15 minutos, que podem ser justificados pela liberação do fármaco de compartimentos de reserva, principalmente dos pulmões (KUIPERS et al., 1999; BESTER, 2009).

Embora os tempos de recuperação não tenham sido um dos parâmetros avaliados neste estudo, pôde-se observar que os animais despertavam sem estímulo externo nos tempos de coleta de 1 a 1,5 horas após o final da infusão, quando as concentrações plasmáticas oscilavam entre 4 e $6 \mu \mathrm{g} \mathrm{ml}^{-1}$. Tamanho (2010) encontrou tempos para decúbito esternal dos animais que variavam de 1,5 a 2 horas após 90 minutos de infusão, utilizando propofol em nanoemulsão e em emulsão lipídica. No entanto, sabe-se que, em gatos, um aumento no tempo de infusão resulta em aumento nos tempos de recuperação (PASCOE et al., 2006).

$\mathrm{Na}$ literatura disponível, não se encontram dados referentes às concentrações plasmáticas efetivas do propofol em felinos, bem como para o despertar anestésico. ADAM et al. (1980), utilizando propofol em uma formulação a base de cremofor, encontraram que concentrações em torno de $4,3 \mu \mathrm{g}$ $\mathrm{ml}^{-1}$ são suficientes para induzir um estado leve de sono em felinos. Entretanto, necessita-se de maiores estudos em felinos, visto que os animais do presente estudo apresentavam concentrações individuais que variavam de 5 a $40 \mu \mathrm{g} \mathrm{ml}^{-1}$ (Figura 1) com planos anestésicos superficiais.

Em relação aos principais parâmetros farmacocinéticos, não houve diferença significativa 

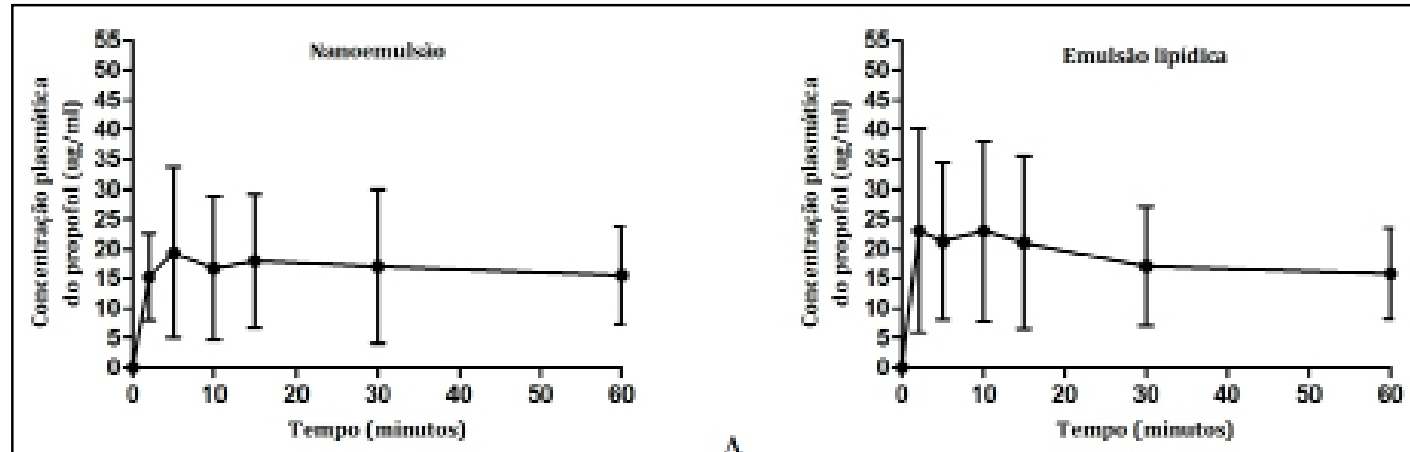

A
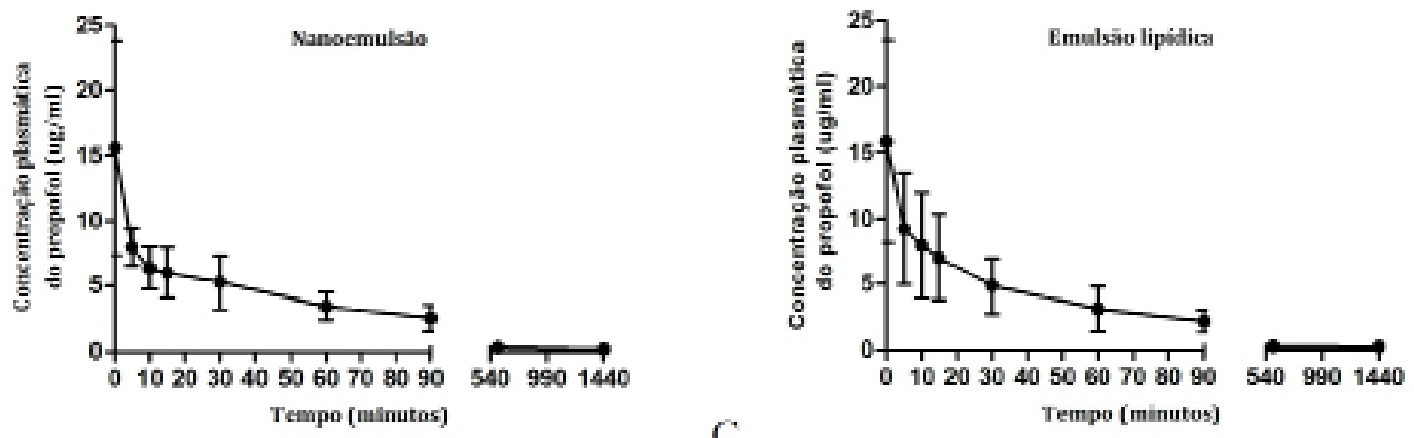

C

Figura 1 - Concentrações plasmáticas $(\mu \mathrm{g} / \mathrm{ml})$ médias e desvios padrão de propofol a 1\% em nanoemulsão e em emulsão lipídica versus tempo (minutos) em gatos $(\mathrm{n}=6)$. A- concentrações de propofol em nanoemulsão durante 60 minutos de infusão; Bconcentrações de propofol em emulsão lipídica durante 60 minutos de infusão; C- curva de decaimento da concentração plasmática de propofol em nanoemulsão após 60 minutos de infusão; D- curva de decaimento da concentração plasmática de propofol em emulsão lipídica após 60 minutos de infusão.

em todos os parâmetros entre as formulações (Tabela 1). A alteração de determinada formulação pode resultar em mudanças em sua farmacocinética e isso ocorre porque os veículos diferentes podem retardar ou acelerar a liberação do fármaco, o que acarretaria alteração das concentrações e da distribuição, ou por competição de sítios metabólicos, que resultaria em diminuição da eliminação (BRAVO GONZÁLES et al., 2004). MOREY et al. (2006) foram pioneiros no desenvolvimento de nanoemulsões para o propofol, desenvolvendo uma a base de polaxamer 188 a $5 \%$ e o sal de ácido graxo a $0,2 \%$, obtendo nanoparticulas de em média $24,5 \mathrm{~nm}$. Os autores não encontraram diferenças em relação à dose e ao efeito anestésico, quando comparada à emulsão comercial. KIM et al. (2007) apresentaram uma nova formulação a base de $8 \%$ de polietilenoglicol, 660 hidroxiesterase (Solutol HS 15), como surfactante não-iônico, e $5 \%$ de tetrahidrofurfuril álcool polietilenoglicol ester como cosurfactante, com partículas de em média 30,9nm. Neste estudo, em humanos, os autores encontraram uma menor distribuição tecidual da nanoemulsão e uma liberação mais rápida do fármaco, resultados do menor tamanho molecular e menor lipossolubilidade da partícula. Porém, essas alterações não resultaram em alterações na dosagem e efeito anestésico. Mais tarde, LEE et al. (2009), em cães, apresentaram uma reformulação da nanoemulsão de KIM et al. (2007) a base de $10 \%$ de polaxamer 188 e $0,7 \%$ de Solutol HS 15 e não encontraram diferenças na farmacocinética e farmacodinâmica do propofol, quando comparado à emulsão lipídica comercial. Os autores atribuem as diferenças em relação ao estudo de KIM et al. (2007) aos diferentes surfactantes, já que o polaxamer 188 e o Solutol HS 15 apresentam elevada distribuição nos compartimentos extracelulares, devido à hidrossolubilidade e tamanho das partículas, o que pode elevar o Vd. 
Tabela 1 - Valores médios e desvios padrão dos parâmetros farmacocinéticos de gatos $(\mathrm{n}-6)$ após bolus de $10 \mathrm{mg} . \mathrm{kg}-1$ min-1 de propofol a $1 \%$ em nanoemulsão ou em emulsão lipídica, seguido de 60 minutos de infusão contínua na taxa de $0,3 \mathrm{mg} \mathrm{kg}^{-1} \mathrm{~min}^{-1}$. Os parâmetros foram calculados a partir da curva de decaimento ao final da infusão

\begin{tabular}{|c|c|c|c|c|}
\hline \multirow[t]{2}{*}{ Parâmetros } & \multicolumn{2}{|c|}{ 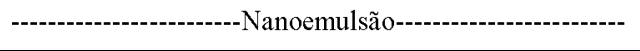 } & \multicolumn{2}{|c|}{---------------------Emulsão lipídica--------------------- } \\
\hline & Média & DP & Média & $\mathrm{DP}$ \\
\hline $\mathrm{T}^{1} / 2 \alpha(\min )$ & 10,20 & 8,80 & 11,30 & 5,62 \\
\hline $\mathrm{T} 1 / 2 \beta(h)$ & 1,34 & 0,26 & 1,24 & 0,46 \\
\hline $\mathrm{T}^{1 / 2 \gamma}(\mathrm{h})$ & 21,52 & 10,34 & 15,69 & 9,21 \\
\hline $\mathrm{ASC} 0-25$ & 18,68 & 6,79 & 17,92 & 7,84 \\
\hline$\triangle \mathrm{SCO}-8$ & 24,59 & 8,76 & 24,24 & 11,91 \\
\hline $\operatorname{MRT}(h)$ & 17,96 & 9,25 & 15,98 & 9,78 \\
\hline $\mathrm{V} 1(1 / \mathrm{kg})$ & 2,57 & 1,02 & 2,72 & 1,62 \\
\hline $\mathrm{V} 2(1 / \mathrm{kg})$ & 2,03 & 1,08 & 2,27 & 1,22 \\
\hline V3 $(1 / \mathrm{kg})$ & 18,63 & 10,98 & 13,14 & 6,56 \\
\hline Vdss $(1 / \mathrm{kg})$ & 23,23 & 12,30 & 18,12 & 8,54 \\
\hline $\mathrm{C} 11\left(\mathrm{ml} \mathrm{kg}^{-1} \mathrm{~min}^{-1}\right)$ & 22,20 & 10,83 & 23,42 & 13,50 \\
\hline $\mathrm{Cl} 2\left(\mathrm{ml} \mathrm{kg}^{-1} \min ^{-1}\right)$ & 141,56 & 125,43 & 74,74 & 51,58 \\
\hline $\mathrm{C} 13\left(\mathrm{ml} \mathrm{kg}^{-1} \mathrm{~min}^{-1}\right)$ & 21,03 & 11,17 & 27,49 & 18,88 \\
\hline $\mathrm{k} 10\left(\mathrm{~h}^{-1}\right)$ & 0,58 & 0,33 & 0,54 & 0,15 \\
\hline$k 12\left(h^{-1}\right)$ & 5,80 & 8,94 & 1,77 & 1,23 \\
\hline$k 13\left(h^{-1}\right)$ & 0,53 & 0,23 & 0,60 & 0,14 \\
\hline$k 21\left(h^{-1}\right)$ & 3,70 & 2,72 & 1,92 & 0,74 \\
\hline $\mathrm{k} 31\left(\mathrm{~h}^{-1}\right)$ & 0,08 & 0,04 & 0,13 & 0,06 \\
\hline
\end{tabular}

A nanoemulsão do presente estudo emprega como único surfactante o Solutol HS15, que é o de escolha para nanoemulsões, pois não interfere na farmacocinética dos fármacos associados (BRAVO GONZÁLES et al., 2004). No registro de patente dessa nova nanoemulsão, os criadores referem partículas que mantêm diâmetro médio inferior a 50nm, mesmo após 12 meses e com alta estabilidade. Embora na patente seja citada uma maior potência da nanoemulsão (cerca de $2 \mathrm{x}$ a da formulação comercial atual), os estudos de TAMANHO (2010), em gatos, com a respectiva formulação, não encontraram diferenças anestésicas, na dosagem ou no tempo de recuperação dos pacientes.

Em gatos, o único estudo farmacocinético com nanoemulsões é o de CLEALE et al. (2009) que, embora também não tenham evidenciado diferenças entre as formulações, usaram uma nanoemulsão diferente das demais, composta por propofol a $1 \%$ em uma formulação aquosa não lipídica a base de $8 \%$ de polaxamer 188 , $3 \%$ de polietilienoglicol $400,1 \%$ de propilenoglicol, $0,2 \%$ de ácido cítrico, $0,18 \%$ de metilparaben, $0,02 \%$ de propilparaben, água e excipientes.

Em relação ao volume de distribuição, puderam-se observar valores altos de V3 e Vdss (Tabela 1), o que demonstra uma elevada distribuição tecidual do propofol. Entretanto, os valores foram maiores que os citados em outros estudos (ADAM et al., 1980; BESTER, 2009; CLEALE et al., 2009). A utilização de infusão contínua pode ter prejudicada a determinação da dose de carga utilizada para cálculo do $\mathrm{Vd}$, diferente dos estudos anteriormente citados, que utilizaram administração em bolus. Ainda, quanto menor o tempo da primeira coleta de amostra, melhor defindo o Vd (BESTER, 2009), o que pode ter superestimado este valor no presente estudo. $\mathrm{O}$ fato de os animais serem castrados pode acarretar em aumento da taxa de gordura corporal, o que pode elevar os valores de V3 e Vdss, já que estes representam principalmente a distribuição nos tecidos de reserva (CORTÍNEZ et al., 2010)

$\mathrm{O}$ tempo de meia vida de distribuição $\left(t_{1 / 2} \alpha\right)$, embora considerado curto, foi maior em relação a outras espécies (ADAM et al., 1980; NOLAN \& REID, 1993). Entretanto, a grande variação individual encontrada também é citada por BESTER (2009). ADAM et al. (1980) demonstraram, em suínos, que a utilização de infusão contínua pode aumentar a t $1 / 2 \alpha$, pois, ao final da infusão já se está no estado de equilíbrio, ocasionando menor transferência do fármaco entre os compartimentos.

As meias vidas de eliminação $\beta$ e $\gamma\left(t_{1 / 2} \beta\right.$ e $t_{1 / 2} \gamma$ ) foram maiores em relação a outras espécies como esperado em felinos, já que estes sabidamente 
são deficientes em metabolizar o propofol. Isso resulta em uma lenta eliminação do fármaco após grandes períodos de infusão. BESTER (2009) utilizou os dados de seu estudo em uma simulação gráfica computacional e observou que, após um período de infusão de 20 minutos, a meia-vida do propofol em gatos tende a aumentar progressivamente, diferente de humanos. TAMANHO (2010), utilizando as mesmas formulações do presente estudo, encontrou tempos de recuperação condizentes com o $\mathrm{t}^{1} / 2 \beta$ deste trabalho.

O $t^{1 / 2} \gamma$ encontrado demonstra o efeito cumulativo do propofol em gatos, devido à lenta eliminação. Isso resultou em um maior tempo de permanência do fármaco no organismo (MRT),

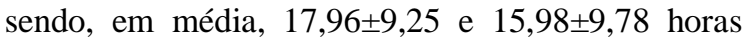
para a nanoemulsão e para emulsão lipídica, respectivamente, demonstrando um efeito cumulativo do fármaco no organismo. PASCOE et al. (2006) corroboram essas afirmações, pois evidenciaram aumento nos tempos de recuperação relacionado com o tempo de duração da infusão do fármaco. GALL et al. (2011) verificaram que a presente nanoemulsão e a emulsão lipídica após repetidas administrações em bolus durante 5 dias podem apresentar efeito cumulativo, porém sem significância clínica.

Em comparação com outras espécies, os valores de clearance foram baixos (Tabela 1), como esperado para felinos (ADAM et al., 1980; CLEALE et al., 2009). Ao comparar os valores obtidos neste estudo com os de BESTER (2009), observaramse valores acima dos encontrados pelo autor, que verificou um $\mathrm{Cl}$ de $8,6 \pm 4,3 \mathrm{~mL} \mathrm{~kg}^{-1} \mathrm{~h}^{-1}$. No entanto, CLEALE et al. (2009) encontraram valores médios próximos a $20 \mathrm{~mL} \mathrm{~kg}^{-1} \mathrm{~min}^{-1}$ tanto para a nanoemulsão quanto para emulsão lipídica, corroborando os dados deste estudo.

Os baixos valores de clearance justificam o aumento nos tempos de meia-vida em relação a outras espécies, como já discutido anteriormente. A deficiência de metabolização de compostos fenólicos já é bem definida em felinos e deve-se ao fato do gene que decodifica a enzima glucuroniltransferase ser classificado como um pseudogene, que ocasiona déficit na formação de compostos glicuronizados (COURT \& GREENBLATT, 2000). Ainda, outros estudos demonstraram maior tempo de recuperação em felinos após administração de propofol (PASCOE et al., 2006; TAMANHO, 2010), correlacionando à lenta biotransformação desse fármaco na espécie.

Ofluxo sanguíneo hepáticoem gatosécitado como em torno de $21 \mathrm{~mL} \mathrm{~kg}^{-1} \mathrm{~min}^{-1}$ (GREENWAY \& OSHIRO, 1972), assim, os valores de clearance do propofol encontrados estão iguais ou abaixo destes, o que pode indicar baixa extração hepática do fármaco. BESTER (2009), supôs que, devido a esta extração deficiente, o débito cardíaco pouco influenciaria na depuração do propofol em felinos, pois a variação do fluxo não seria significativa. Mesmo que os valores do presente estudo sejam superiores àqueles encontrados por BESTER (2009), ao associar com os dados de TAMANHO (2010), o qual encontrou maior depressão hemodinâmica em felinos tratados com a emulsão lipídica do que com a nanoemulsão, podese verificar que o autor não encontrou diferença nos tempos de recuperação dos animais, logo, a depressão hemodinâmica não interferiu no metabolismo do fármaco. Por fim, KUIPERS et al. (1999) e BESTER (2009) afirmaram que a extração pulmonar de propofol é alta, em torno de $61 \%$, e que talvez este órgão exerça grande contribuição na metabolização nesta espécie.

\section{CONCLUSÃO}

De acordo com a metodologia proposta, conclui-se que a farmacocinética do propofol em nanoemulsão não difere da emulsão lipídica após infusão contínua em gatos e caracteriza-se por extensa distribuição tecidual e meia vida de eliminação terminal prolongada, o que pode resultar em efeito cumulativo.

\section{COMITÊ DE ÉTICA E BIOSSEGURANÇA}

Este estudo foi aprovado pelo Comitê de Ética e Bem Estar Animal (CEBEA) da instituição de origem, sob o protocolo $1.36 / 10$.

\section{REFERÊNCIAS}

ADAM, H.K. et al. Pharmacokinetics in laboratory animals of ICI 35 868, a new i.v.anaesthetic agent. British Journal of Anaesthesia, v.52, p.743-746, 1980.

BAKER,M.T.;NAGUIB,M. Propofol:thechallenges of formulation. Anesthesiology, v.103, p.860-876, 2005. Disponível em: <http:// graphics.tx.ovid.com/ovftpdfs/FPDDNCDCDGMIDI00/fs046/ ovft/live/gv025/00000542/00000542-200510000-00026.pdf >. Acesso em: 20 fev. 2011.

BESTER, L. Pharmacokinetics of propofol in cats. 2009. 72f. PhD Dissertation, Department of Companion Animal Clinical Studies, Faculty of Veterinary Science, University of Pretoria, Pretoria, South Africa.

BRAVO GONZÁLEZ, R.C. et al. In vitro investigation on the impact of Solutol HS15 on the uptake of colchicine into rat hepatocytes. International Journal of Pharmaceutics, v.279, p.27-31, 2004. Disponível em: <http://dx.doi.org.ez74.periodicos.capes.gov. br/10.1016/j.ijpharm.2004.04.010>. Acesso em: 12 nov. 2011.

CLEALE, R.M. et al. Pharmacokinetic and pharmacodynamic evaluation of propofol administered to cats in a novel, aqueous, 
nano-droplet formulation or as an oil-in-water. Journal of Veterinary Pharmacology and Therapeutics, v.32, p.436445, 2009. Disponível em: <http://dx.doi.org/10.1111 /j.1365-2885.2009.01059.x>. Acesso em: 12 fev. 2011.

COURT, M.H.; GREENBLATT, D.J. Molecular genetic basis for deficient acetaminophen glucuronidation by cats: UGT1A6 is a pseudogene, and evidence for reduced diversity of expressed hepatic UGT1A isoforms. Pharmacogenetics, v.10, p.355-369, 2000

CRISTÁLIA PRODUTOS QUÍMICOS FARMACÊUTICOS LTDA, São Paulo-BR, Ogari de Castro Pacheco, Roberto Debom Moreira, Marisa Dutra Rodrigues Rizzi. Microemulsão óleo/água de propofol estável e pronta para uso. N. PI 0604377-1 A. BR. 27 Out. 2006. 24 Jun. 2008. República Federativa do BrasilMinistério do Desenvolvimento, Indústria e do Comércio ExteriorInstituto Nacional da Propriedade Industrial.

DATE, A.A.; NAGARSENKER, M.S. Design and evaluation of microemulsions for improved parenteral delivery of propofol. American Association of Pharmaceutical Scientists, v.9, p.138145, 2008a. Disponível em: <http://dx.doi.org/10.1208/s12249007-9023-7>. Acesso em: 2 nov. 2011.

GALL, G.O. et al. Efeito cumulativo do propofol em nanoemulsão e em emulsão lipídica em gatas. In: ENCONTRO DE ANESTESIOLOGIA VETERINÁRIA, 10., 2011, Campos do Jordão, SP. Anais... Campos do Jordão: 1 CD-ROM, 2011. p.15.

GREENWAY, C.V.; OSHIRO, G. Comparison of the effects of hepatic nerve stimulation on arterial flow, distribution of arterial and portal flows and blood content in the live of anaesthetized cats and dogs. Journal of Physiology, v.227, p.487-501, 1972.

KIM, K.M. et al. Pharmacokinetics and pharmacodynamics of propofol microemulsion and lipid emulsion after an intravenous bolus and variable rate infusion. Anesthesiology, v.106, p.924-934, 2007. Disponível em: <dx.doi.org/10.1097/01. anes.0000265151.78943.af>. Acesso em: 2 fev. 2011.

KUIPERS, J.A. et al. First-pass lung uptake and pulmonary clearance of propofol: Assessment with a recirculatoryindocyanine green pharmacokinetic model. Anesthesiology, v.91, p.17801787, 1999 .

LEE, S.H. et al. Pharmacokinetics and pharmacodynamics of a new reformulated microemulsion and the long-chain triglyceride emulsion of propofol in beagle dogs. British Journal of Pharmacology, v.158, p.1982-1995, 2009. Disponível em: <http://onlinelibrary.wiley.com.ez74.periodicos.capes.gov.br/doi/1 0.1111/j.1476-5381.2009.00509.x>. Acesso em: 2 fev. 2011.

MOREY, T.E. et al. Anesthetic properties of a propofol microemulsion in dogs. Anesthesia and Analgesia, v.103, p. 882887, 2006b. Disponível em: <http://www.ncbi.nlm.nih.gov/pmc/ articles/PMC2999695/?tool=pubmed>. Acesso em: 08 ago. 2012.

NOLAN, A.; REID, J. Pharmacokinetics of propofol administered by infusion in dogs undergoing surgery. British Journal of Anaesthesia, v.70, p.546-551, 1993.

PASCOE, P.J. et al. The effect of the duration of propofol administration on recovery from anesthesia in cats. Veterinary Anaesthesia and Analgesia, v.33, p.2-7, 2006. Disponível em: <http://onlinelibrary.wiley.com.ez74.periodicos.capes.gov.br/doi/1 0.1111/j.1467-2995.2005.00216.x>. Acesso em: 2 fev. 2011.

TAMANHO, R.B. Efeitos cardiorespiratórios e metabólicos do propofol nas formulações em emulsão lipídica e nanoemulsão em felinos. 2010. 120f. Dissertação (Mestrado em Ciência Animal) - Centro de Ciências Agroveterinárias, Universidade do Estado de Santa Catarina, Lages, SC. 